\title{
Aprender Seguros: Escuelas que Cuidan
}

\section{Un programa para fortalecer los vínculos en la escuela}

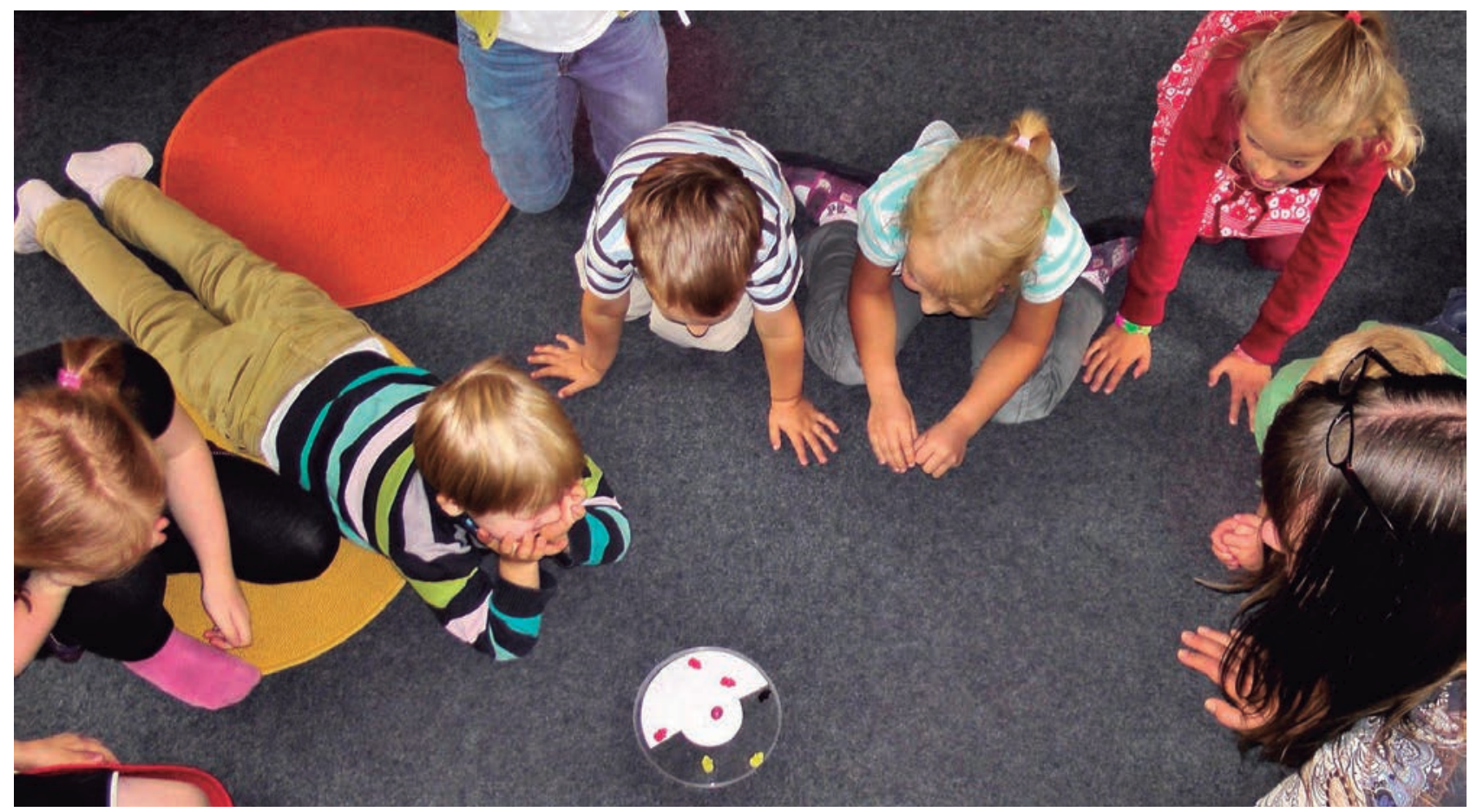

La seguridad es clave para que el aprendizaje pueda llegar a darse $y$, en los primeros años de vida, esta viene dada por quienes nos acompañan y nos cuidan, por nuestras figuras de referencia. El programa Aprender Seguros: Escuelas que Cuidan forma a los profesionales del ámbito escolar para asegurar una transmisión descendente de los cuidados donde los docentes puedan ofrecer seguridad a los alumnos y sus familias $y$, a su vez, puedan recibir seguridad de quienes los

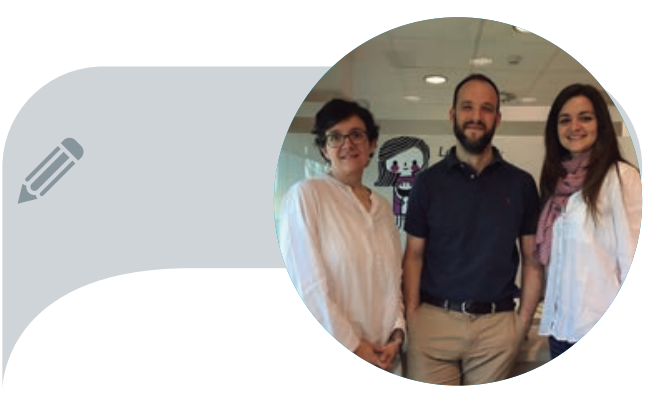

Amaia Halty Universidad Pontificia Comillas ahalty@comillas.edu

Carlos Pitillas Universidad Pontificia Comillas

cpitillas@comillas.edu

Ana Berástegui Universidad Pontificia Comillas a.berastegui@comillas.edu coordinan o dirigen. 


\section{La importancia del vínculo para el desarrollo integral del alumno}

El alumno necesita sentirse suficientemente seguro para aprender. Por ello, una dimensión fundamental de la escuela, cuyo objetivo es el desarrollo integral de sus alumnos, será lograr ofrecerle esa seguridad. La seguridad permite que el cerebro del alumno despliegue, por ejemplo, una mayor atención y se involucre más en la tarea que el cerebro de un alumno que se siente inseguro. Este último va a estar más centrado en detectar aquello que le genera inseguridad y en tratar de encontrar los medios para salir de esa situación de alerta o de "peligro". La evolución de miles de años ha hecho que nuestro cerebro prime la supervivencia frente a cualquier otra tarea y es por ello por lo que la vivencia de seguridad es fundamental para que la apertura hacia lo nuevo, a la exploración, a la aventura, etc., puedan darse.

\section{¿Cómo se consigue la seguridad en un alumno?}

Desde el comienzo de la vida extrauterina, el bebé viene programado para buscar la seguridad en sus figuras de cuidado (habitualmente sus padres). El bebé necesita ser regulado en su temperatura, alimentación, higiene, sueño y en su percepción de seguridad. Si el bebé se siente desregulado en alguna de estas necesidades básicas, comenzará a emitir conductas como el llanto, poner caras de desagrado, agitarse motrizmente, etc., con el fin de hacer saber a sus cuidadores que necesita que lo regulen. Si los cuidadores son capaces de responder adecuadamente a las necesidades del bebé estarán construyendo un sentimiento de seguridad en los otros y también en sí mismo "me entienden, me hago entender", "me dan lo que necesito para estar bien, consigo pedir lo que necesito". Asimismo, el bebé también viene programado para aprender, para explorar su entorno y poner a prueba sus capacidades. Para el despliegue de todo ello, también necesita que sus figuras de cuidado lo acompañen, estimulen y protejan, es decir, le ofrezcan

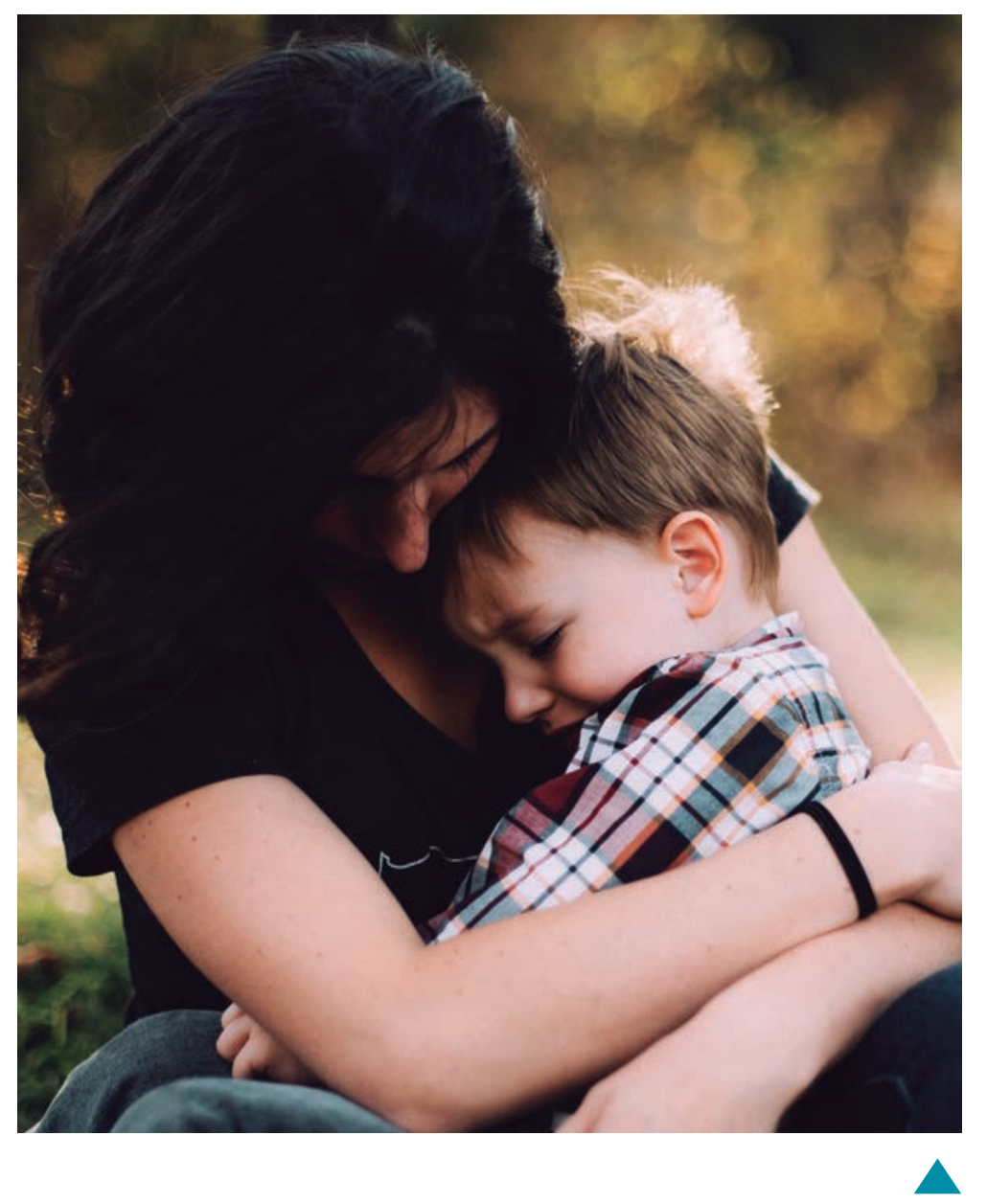

seguridad. Cuanto más seguro y confiado se sienta el bebé en su aventura de conocer e interactuar con el mundo exterior, de "salir hacia fuera", más tiempo podrá permanecer en ella y, por tanto, desarrollar habilidades y competencias.

Así, la percepción de seguridad en el bebé va a venir dada por cómo sus figuras de cuidado se hacen cargo de sus necesidades, tanto las de ser regulado emocional y biológicamente, como las de sentirse acompañado y respaldado en la exploración. Puede decirse entonces que la seguridad del niño se construye a través de la relación con sus figuras de cuidado, lo que desde la teoría del apego se denomina vínculo de apego. La multitud de microinteracciones que se producen diariamente entre el bebé y sus padres van generando en el primero una mayor o menor percepción de seguridad; un vínculo de apego más o menos seguro. Este cúmulo de experiencias a lo largo de sus primeros años de vida irá conformando en él una imagen de sí mismo (¿quién soy yo? ¿Soy alguien digno de ser querido? ¿Soy alguien que vale la pena? ¿Merezco ser atendido?), de los demás (¿puedo confiar 
La seguridad es fundamental para la creación de una buena imagen de uno mismo, de las propias capacidades y de la confianza en los demás

en los demás?), del entorno (¿me ocurren cosas positivas cuando salgo a explorar? ¿El mundo que percibo es un lugar seguro?) y de sus capacidades (¿puedo obtener lo que necesito? ¿Soy capaz de hacerme entender?). La respuesta a estas preguntas lo acompañará, como un guion, no siempre consciente, al encuentro de nuevas relaciones.

A veces con algunos años de vida y otras con tan solo algunos meses, los niños o bebés acceden a la escolarización infantil. De hecho, los últimos datos publicados por el INE nos indican que más del 40\% de los niños de un año, y más del 95\% de los niños de 3 años estaban escolarizados en el curso 2017-2018. Sin duda, el tiempo que pasan en la escuela va a modular su percepción de seguridad y va a contribuir a la construcción de su propia imagen, de sus capacidades, de su percepción del entorno y de los demás. Por ello es tan importante que en la escuela se cuide y se ponga en valor no solo el acompañamiento en la exploración y el aprendizaje de nuevas habilidades y destrezas, sino también la regulación emocional de los alumnos. Regular y atender su mundo emocional no solo permite que estén mejor predispuestos al aprendizaje, porque se sientan suficientemente seguros, sino que además les permite comprender cómo funciona su propio mundo emocional y el de los demás, así como confiar en que pueden pedir y recibir ayuda, todo ello cimientos fundamentales del autoconcepto, la autorregulación, la empatía o las relaciones interpersonales.

Los maestros de infantil saben bien lo importantes que son los primeros días de colegio para generar un buen vínculo entre el alumno y ellos. Esas primeras semanas de curso que suelen denominarse "periodo de adaptación" son claves para conseguir que el alumno confíe en sus nuevas figuras de referencia y sentirse así suficientemente seguros para disfrutar de todas las posibilidades que les ofrece la escolarización. Sin embargo, ni todos los niños se sienten igual de seguros pasado este periodo de adaptación, ni la seguridad depende únicamente de estas primeras semanas. A lo largo de una jornada escolar, son muchos los momentos en que uno, varios, o casi todos los niños, pueden sentirse inseguros, desregulados, y activar su sistema de alarma como, por ejemplo, despedirse de sus padres, un empujón de un compañero, quedarse "atascado" en una tarea, perder de vista a su profesor, salir al patio o cambiar de aula, un ruido inesperado, un material nuevo, ver a un compañero llorar, estar cansado, enfermo o con hambre, etc.

\section{¿Cómo promovemos la seguridad en el aula?}

Cuando entras en un aula de infantil $y$ ves que hay varios niños desregulados (algunos llorando y otros tristes, enfadados, tirando de la bata de la profesora o gritando "mamáááá"), y al cabo de 5-10 minutos esa clase se ha convertido en pura armonía (con todos los alumnos jugando, disfrutando o mostrando entusiasmados sus avances a la profesora) sientes que algo parecido a la magia ha sucedido en esa clase. La profesora ha sido capaz de detectar las necesidades de cada alumno y satisfacerlas adecuadamente, proporcionando un ambiente de seguridad que revierte en todo el alumnado. Desde ASEC promovemos un entrenamiento de la mirada que permita a los educadores detectar y comprender con mayor facilidad las necesidades subyacentes a la conducta de sus alumnos para responder de forma más ajustada y eficaz.

Sin embargo, en estas situaciones, no solo es importante lo que la profesora es capaz de hacer en la interacción con 
sus alumnos, sino que también tiene una gran repercusión cómo se siente la profesora en ese momento: ¿está tranquila y confiada o, por el contrario, está nerviosa o alterada? Por ello, además de trabajar sobre lo que ocurre en la interacción con los alumnos, nuestro programa también aborda lo que le ocurre al profesor internamente. En este sentido, en el marco de Primera Alianza trabajamos con la premisa de que "nadie puede dar lo que no tiene" y, por tanto, uno no puede ofrecer seguridad si se siente inseguro o desregulado, es decir, si está nervioso, irritado, enfadado, cansado, etc. Así, destinamos parte de la formación a que los profesores conozcan y detecten con mayor facilidad cuáles son las situaciones que individualmente alteran su mundo interno, para que puedan establecer estrategias eficaces en el aula que les permitan seguir ofreciendo seguridad.

\section{Cuidar la relación familia-escuela hoy}

Desde el ámbito escolar se sabe que la relación que los padres establecen con sus hijos influye notablemente en cómo estos se comportan en la escuela, con los profesores y los compañeros. Asimismo, los profesores también saben y experimentan que la seguridad generada en el ámbito educativo puede ser transformadora para muchos niños, incluso sanadora para aquellos que vienen de entornos familiares de mayor inseguridad.

Cada uno de estos ámbitos relacionales influyen mucho sobre el niño, pero también lo hace la interacción entre ellos. La relación que se establece entre la familia y la escuela influye de forma significativa sobre la seguridad del alumno. Por ello, desde ASEC, se destina un módulo formativo a trabajar sobre esta relación con el objetivo fundamental de generar entre ambas partes alianzas de cuidado que fortalezcan y apoyen la labor de cada una.

Las familias de hoy son habitualmente familias de doble empleo donde las dificultades y, a veces, el estrés por compatibilizar la vida familiar y laboral afectan a su disponibilidad para responder a las

\section{Un buen vínculo ofrece} seguridad. La seguridad permite que nos atrevamos a explorar el mundo y que nos permitamos pedir ayuda, consuelo y afecto cuando lo necesitamos

peticiones del centro educativo o asistir, por ejemplo, a reuniones o eventos. Además, se encuentran inmersas en una cultura de la inmediatez donde se nos facilita e incita a adquirir cualquier tipo de producto (digital, material, alimenticio, etc.) de forma inmediata; y del bienestar, donde se busca en todo momento la felicidad del individuo y se tienden a omitir - rechazar los procesos de sufrimiento, esfuerzo o fracaso. Asimismo, la sobreinformación actual sobre crianza, su accesibilidad y la disminución de redes de apoyo con quienes compartir la crianza, genera en algunos padres desconcierto e inseguridad sobre cómo educar. Estos y otros elementos de nuestro momento social actual influyen sobre los procesos educativos entre padres e hijos y a veces chocan con los tiempos y criterios educativos que se manejan en la escuela.

Por otro lado, la mayoría de los docentes cree y apuesta cada vez más por una atención individualizada del alumno que requiere una mayor dedicación y esfuerzo en su labor docente. Además, la cultura de la inmediatez también les exige estar al día de las nuevas técnicas y herramientas que se desarrollan para el ámbito educativo, así como atender a las familias que les demandan asesoría en cuestiones de crianza y muy especialmente a las familias cuyo hijo o ellas mismas presentan alguna dificultad. Además de encontrarse en una situación actual de sobreexigencia, es importante recalcar que, en su trayectoria formativa, rara vez están incluidas asignaturas o cursos destinados al abordaje de la relación familia-escuela, a pesar de la importancia capital que esta 


\section{IIII. ÁQORA DE DROFESORES}

\section{ALGUNAS PREGUNTAS PARA REFLEXIONAR SOBRE LA SEGURIDAD EN EL AULA}

Desde la teoría del apego, se pueden clasificar las necesidades de los niños en dos grandes categorías: la necesidad de apego y la necesidad de explorar. La necesidad de apego se activa frente a cualquier situación de pérdida de regulación fisiológica 0 emocional y, por tanto, el niño necesita en ese momento que lo calmen, expliquen, cuiden, abracen, alimenten, etc. La necesidad de explorar se activa cuando el niño quiere interactuar con el mundo, en el sentido más amplio de la expresión, por ejemplo, cuando desea jugar, realizar nuevas acciones, separarse de sus figuras de referencia, contactar con otros niños, tocar o saborear objetos, caminar, subirse a los columpios, etc. Ambas necesidades son universales y se manifiestan de forma alterna, es decir, 0 se tiene activada la necesidad de apego 0 se tiene activada la necesidad de explorar. Lo que sí es variable es el tiempo que cada niño tiene activada cada una de las necesidades y cómo cada uno manifiesta su necesidad. No todos los niños manifiestan de la misma forma que quieren explorar o que se sienten desregulados.

Te invitamos a reflexionar sobre estas dos grandes necesidades:

$\checkmark \quad$ ¿Cómo me manifiestan mis alumnos sus necesidades de explorar? ¿Y sus necesidades de apego?

\ ¿Cuál de estas dos necesidades me cuesta más atender en general?

\ ¿Hay algún alumno al que le cueste especialmente salir a explorar? ¿Cómo crees que puedes ofrecerle seguridad para que viva la exploración con éxito?

У ¿Hay algún alumno al que le cueste especialmente pedir ayuda, mostrar cercanía o necesidad de afecto? ¿Cómo crees que puedes mostrarle a ese alumno que puede confiar en ti para que le ayudes a regularse emocionalmente?

\section{En un centro educativo se} transmite seguridad en la medida en que se garantiza una transmisión descendente de los cuidados, donde cada eslabón de la cadena tiene recursos para ofrecer seguridad y para pedirla en caso necesario tiene en su función como docentes, y ello puede hacerles sentir inseguros a la hora de acometer esta tarea.

Esta realidad social descrita con pinceladas, nos asoma algunos de los retos actuales a los que se enfrentan familia y escuela, pero también nos ofrecen un rango amplio de oportunidades sobre las que trabajar y sobre las que poder recibir apoyo mutuo.

Desde ASEC consideramos que el objetivo de la relación familia-escuela es la obtención de una alianza de cuidados simétrica, sin embargo, también creemos que para alcanzar este objetivo la escuela debe inicialmente romper dicha simetría en favor de las familias y colocarse, como entidad profesionalizada, en un rol de ayuda que ofrece seguridad a las familias. Este trabajo podrá desarrollarse en la medida en que los maestros se sientan seguros y competentes para esta labor y, por ello, el segundo módulo formativo va destinado a trabajar sobre claves relacionales generales que ayudan a comenzar la relación "con buen pie", así como abordar y entrenar específicamente algunas situaciones cotidianas que suceden en la interacción con las familias como las tutorías iniciales, las "situaciones de pasillo" o las tutorías "difíciles", todo ello desde una perspectiva de seguridad relacional.

\section{La importancia de la seguridad desde el acompañamiento institucional}

Las personas somos capaces de transmitir seguridad en la medida en que nosotros mismos nos sentimos suficientemente seguros. Por ello, en nuestro deseo de fortalecer la seguridad relacional en los centros educativos, no puede faltar un módulo formativo destinado al manejo y acompañamiento que los coordinadores o directores de centro realizan con sus equipos educativos. En este sentido, desde ASEC, abogamos por establecer y fortalecer cadenas de seguridad en las escuelas, de tal forma que los profesores ofrezcan seguridad a sus alumnos y a las familias de estos, y que los coordina- 


\section{El objetivo no es contar con docentes inquebrantables, sino más bien encontrar las formas de acompañar los momentos de vulnerabilidad para reactivar la capacidad educadora}

dores o directores ofrezcan seguridad a sus equipos docentes. Cuanto más robustas sean estas cadenas, más factible será que el alumno perciba la seguridad que necesita. El objetivo no es contar con docentes inquebrantables, sino más bien encontrar las formas de acompañar los momentos de vulnerabilidad para reactivar la capacidad educadora.

El tercer módulo formativo de ASEC se centra en encontrar maneras de trabajar que aseguren una transmisión descendente de los cuidados, resignificando y reencuadrando, desde una perspectiva de las relaciones seguras, muchas de las habilidades que requieren los cargos de gestión (como ejercer liderazgo, fomentar la autonomía de los trabajadores, ser firme, empático, motivar a los trabajadores, ejercer una escucha activa, posibilitar el desarrollo profesional, gestionar adecuadamente los conflictos, dar confianza, saber celebrar y reconocer los logros, etc.).

Asimismo, en este tercer módulo también se reflexiona con los directivos de

\section{3) Hemos hablado de}

\section{Apego; seguridad; aprendizaje; familia; directivos.}

Este artículo fue solicitado por PADRES Y MAESTROS en mayo de 2020, revisado y aceptado en octubre de 2020.

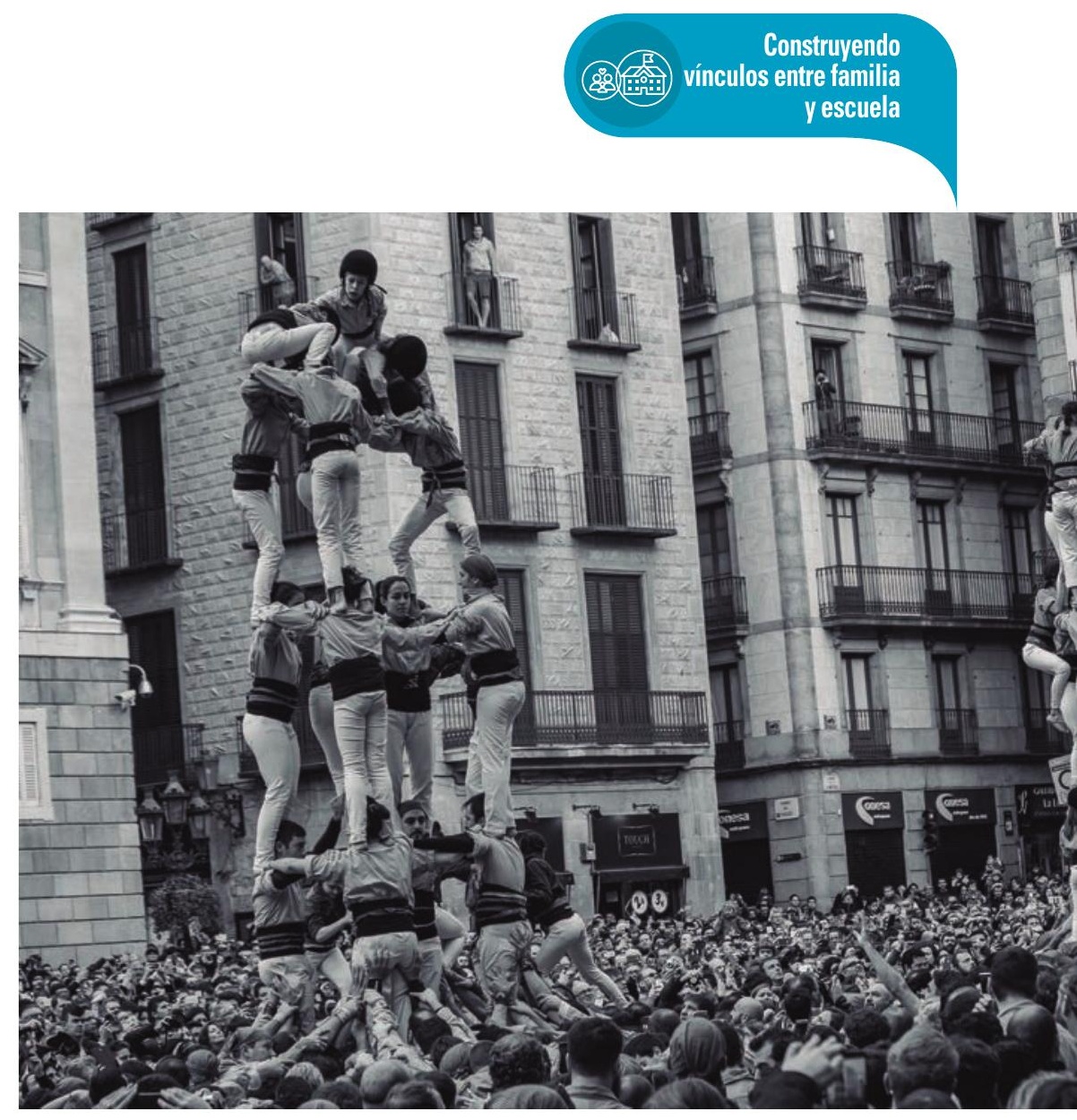

qué manera la seguridad atraviesa no Castellets solo las diferentes relaciones que pueden establecerse en el centro, sino también los espacios en los que esas relaciones se dan y, sobre todo, el currículo del centro educativo. Esta reflexión permite reconocer cómo el currículo (tanto explícito como implícito) puede impregnarse a todos los niveles de una cultura del cuidado que enseña a los más pequeños a cuidar ofreciendo seguridad, tal y como ellos la reciben, así como a cuidar a todos los eslabones que forman parte del engranaje educativo de una escuela $•$

\section{DAR DRR SABER MÁS}

Berástegui, A., Halty, A. \& Pitillas, C. (en prensa). Aprender Seguros: Escuelas que Cuidan. Madrid: Narcea.

Connolly P., Miller, S., Kee, F., Sloan, S., Gildea, A., Mcintosh, E., Boyer, N., \& Bland, M. (2018). A cluster randomised controlled trial and evaluation and costeffectiveness analysis of the Roots of Empathy schools-based program for improving social and emotional well-being outcomes among 8- to 9-year-olds in Northern Ireland. Public Health Research, 6(4). DOI: https://doi. org/10.3310/phr06040

Pitillas, C. \& Berástegui, A. (2018). Primera Alianza: fortalecer y reparar los vínculos tempranos. Barcelona: Gedisa. 